NASA Technical Memorandum 103152

\title{
A Fiber-Optic Current Sensor for Aerospace Applications
}

Richard L. Patterson

Lewis Research Center

Cleveland, Ohio

\begin{abstract}
A.H. Rose, D. Tang, and G.W. Day
National Institute of Standards and Technology

Boulder, Colorado
\end{abstract}

Prepared for the

25th Intersociety Energy Conversion Engineering Conference cosponsored by the AIChE, SAE, ACS, AIAA, ASME, and IEEE

Reno, Nevada, August 12-17, 1990

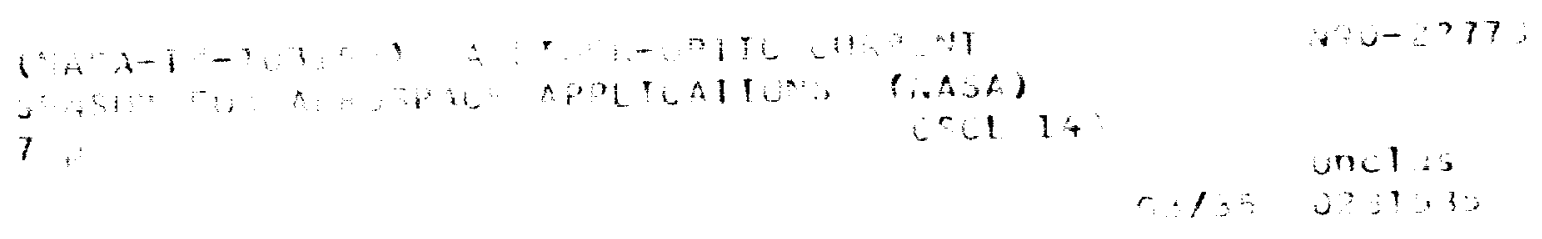

\section{Nons}





\title{
A FIBER-OPTIC CURRENT SENSOR FOR AEROSPACE APPLICATIONS
}

\author{
Richard L. Patterson \\ NASA Lewis Research Center \\ Cleveland, Ohio 44135 \\ A. H. Rose, D. Tang and G. W. Day \\ National Institute of Standards and Technology \\ Boulder, Colorado 80303
}

\begin{abstract}
A robust, accurate, broad-band, alternating current sensor using fiber optics is being developed for space applications at power frequencies as high as $20 \mathrm{kHz}$. It can also be used in low and high voltage $60 \mathrm{~Hz}$ terrestrial power systems and in $400 \mathrm{~Hz}$ aircraft systems. It is intrinsically EMI immune and has the added benefit of excellent isolation.

The sensor uses the Faraday effect in optical fiber and standard polarimetric measurements to sense electrical current. The primary component of the sensor is a specially treated coil of single-mode optical fiber, through which the current carrying conductor passes. Improved precision is accomplished by temperature compensation by means of signals from a novel fiberoptic temperature sensor embedded in the sensing head.

This paper reports on the technology contained in the sensor and also relates the results of precision tests conducted at various temperatures within the wide operating range. It also shows the results of early EMI tests.
\end{abstract}

\section{INTRODUCTION}

The National Institute of Standards and Technology at Boulder and the NASA Lewis Research Center are developing a fiber optic alternating current sensor for space applications. The sensor is designed to have good accuracy with the added benefit of excellent isolation. As a special payoff, it can be used to reduce the electrical upset effects of static discharge in spacecraft and EMI (Electro-Magnetic Interference) in space systems. Because the sensor is broad-banded, it can function well with high frequency $(20 \mathrm{kHz})$ power systems being developed for lunar bases, advanced launch vehicles, orbiting spacecraft, and for travel to Mars. It may be valuable for new motor controllers which use high frequency power. Additionally, it can provide an isolated method to measure current in high voltage $60 \mathrm{~Hz}$ terrestrial power systems, and it can enable EMI immune current measurement in $400 \mathrm{~Hz}$ aircraft systems (especially important when new composite structures will reduce the amount of EMI shielding that metal wings and structures provided).

In this paper, we discuss the fiber-optic current sensor technology that has been developed, and we relate the results of accuracy and environment tests.

\section{SENSOR TECHNOLOGY}

\section{Faraday Effect}

A schematic diagram of the sensor is shown in Figure 1. The sensor uses the Faraday effect in a singlemode fiber. The Faraday effect is a rotation of the plane of polarization of light as it propagates through a material in the direction of a magnetic field; it is proportional to the integral of the magnetic field along the direction of propagation. The sensing element consists of a coil of fiber around the conductor. Since the integral of the magnetic field around a conductor is proportional to the current flowing through it, the rotation of the plane of polarization is proportional to the current and independent of stray fields. Using multiple turns of fiber increases the sensitivity. The constants of proportionality are the magnetic permeability $\mu$ of the material, a material parameter $V$ (known as the Verdet constant), and the number of turns of fiber $\mathbf{N}$.

Linearly polarized light is transported from the laser to the sensing coil by a polarization maintaining (PM) 
fiber. The light with its rotated state of polarization is transported back along a PM fiber and through a polarizing beam splitter to sensing photodiodes. The rotation of the polarization state is converted by these optical elements into a change in transmittance so that a direct measure of the current in the conductor can be made [1].

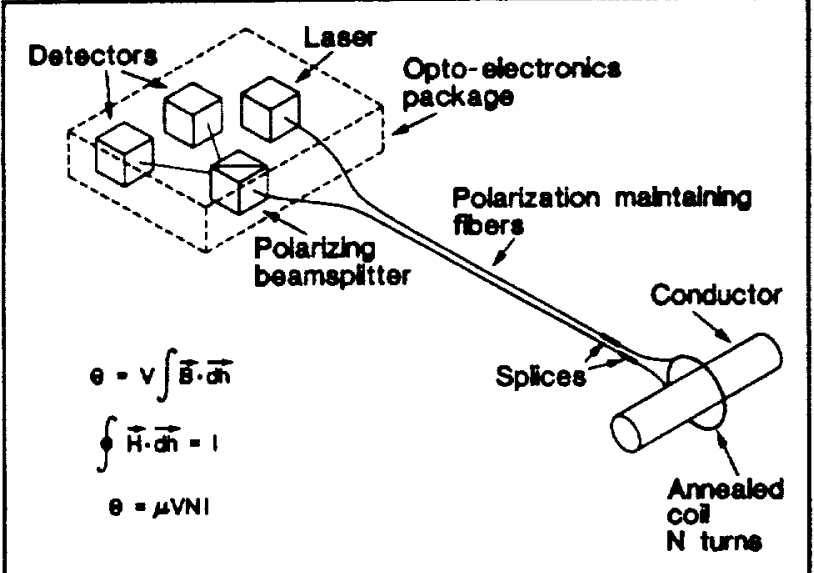

Figure 1 Fiber-Optic Current Sensor.

The Verdet constant in glass varies with both wavelength and temperature. It is approximately inversely proportional to the square of the wavelength. For $0.1 \%$ stability, this requires an optical source that is stable to approximately $\pm 0.4 \mathrm{~nm}$. The normalized temperature dependence of the Verdet constant of $\mathrm{SiO}_{2}$ is about $0.7 \times 10^{-4} /{ }^{\circ} \mathrm{C}[2]$. Therefore, temperature compensation is required if the sensor is to hold its calibration to $\pm 1 \%$ over a temperature range greater than $150^{\circ} \mathrm{C}[3]$.

\section{Linear Birefringence}

Linear birefringence in the optical fiber coil diminishes the Faraday effect [1,3,4]. (A linearly birefringent optical fiber is one in which two orthogonal linear polarization states propagate with slightly different phase velocities.) Linear birefringence may be present in the fiber because of inherent geometrical imperfections and stress resulting from the manufacturing process. It will also result from bending the fiber into a coil.

The inherent birefringence in a single-mode fiber can be nearly eliminated with manufacturing methods that have been developed during the last decade [4]. Bend birefringence remains a problem.

Several approaches to solving the problem of bend birefringence can be considered $[5,6,7,8]$. Our approach is to remove the linear birefringence of the coil by annealing. This approach has been successfully applied to coils ranging from $5 \mathrm{~mm}$ to $10 \mathrm{~cm}$ in diameter with 200 or more turns $[9,10]$. Figure 2 shows an optical fiber coil which has been processed in an annealing cycle and which then holds its coil shape, without restraint. The coils do not retain their protective coating after annealing, and must be packaged in a way that preserves their low linear birefringence.

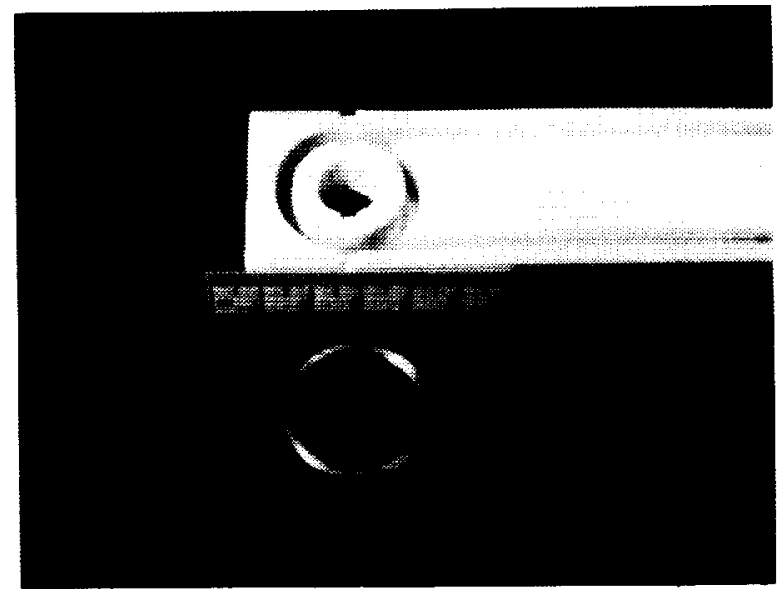

Figure 2 An Optical Fiber Coil after Annealing.

Coil packaging has produced desired results over a broad temperature range, though at low temperatures, packaging remains a problem. Figure 3 shows the measured linear birefringence of a $5 \mathrm{~cm}$ diameter, 50 turn annealed coil as a function of temperature. The coil was embedded in a high viscosity lubricant containing Teflon to protect it and isolate it from vibration. The change in birefringence with temperature, at temperatures above about $-10^{\circ} \mathrm{C}$ seems to be an inherent fiber property, but at lower temperatures the birefringence increases rapidly, apparently as a result of stress applied due to solidification of the lubricant. (New lubricants are now being sought to eliminate the solidification problem.) As a consequence of the temperature dependence of the residual birefringence, the sensitivity of the sensor above $-10^{\circ} \mathrm{C}$ showed a temperature coefficient of approximately $1.7 \times 10^{-4} /{ }^{\circ} \mathrm{C}$. 


\section{ORIGINAL PAGE \\ BLACK AND WHITE PHOTOGRAPH}

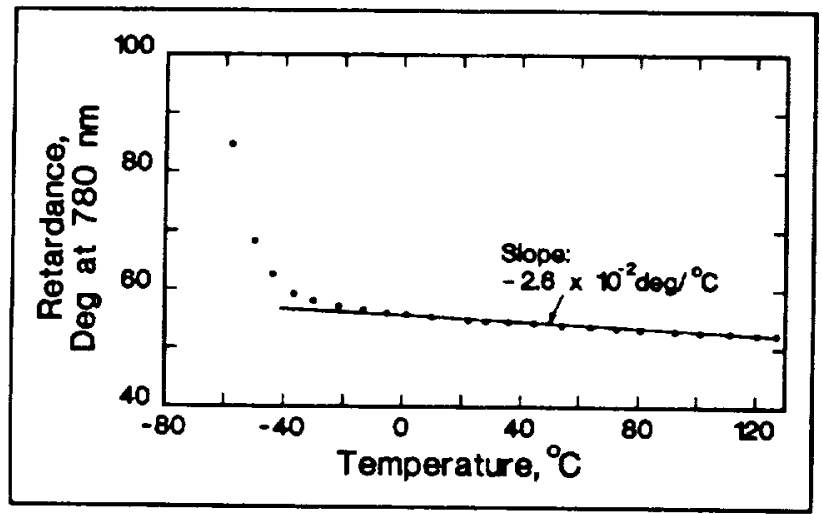

Figure 3 Birefringence as a Function of Temperature.

\section{Opto-electronics Package}

At present, the opto-electronics package has been designed to operate near room temperature. In the future, an opto-electronics box will be designed to operate over a broader temperature range approaching $-65^{\circ} \mathrm{C}$ to $125^{\circ} \mathrm{C}$

To achieve an acceptable sensitivity we need a sufficient amount of optical power at a relatively short wavelength. These considerations, along with the need for a rugged and mechanically stable source led to the use of a semiconductor laser at a wavelength of $780 \mathrm{~nm}$. Since the source needs to be well polarized and have a spectrally broad output to avoid coherence effects, we chose a multi-longitudinal mode laser (with a polarization extinction ratio of about $10 \mathrm{~dB}$ ) followed by a polarizer to improve the extinction ratio to about 30 $\mathrm{dB}$.

Figure 4 shows the laser package. The required optical elements are within the aluminum package; a thermoelectric heater/cooler (beneath the laser package and the baseplate) is used to control the temperature.

Silicon PIN photodetectors are the most suitable for high stability applications in this wavelength region. Although their responsivity depends on wavelength, it varies only slightly with temperature. However, both of these effects are, in principle, compensated by normalizing the output signal to the average transmitted power.

Although the sensing element is constructed entirely of dielectric materials, and therefore can be placed in a high EMI environment without difficulty, the sources and detectors are particularly prone to interference and must be well shielded.

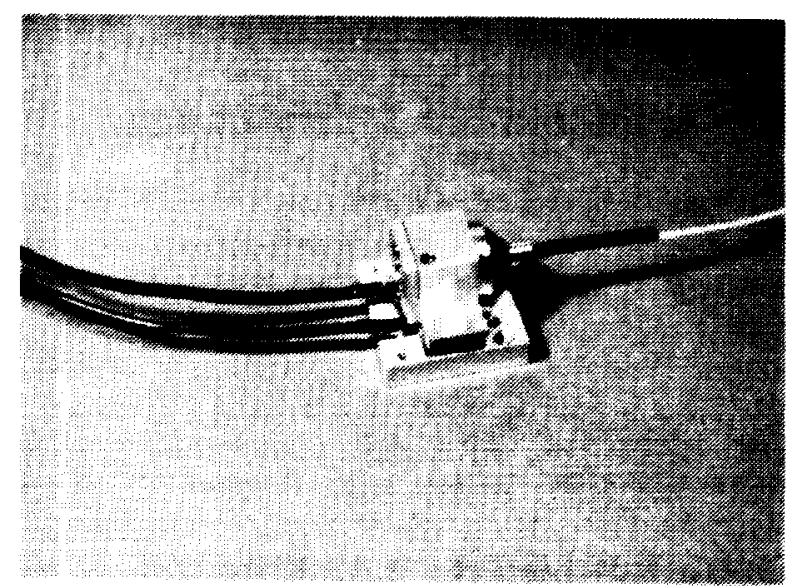

Figure 4 Thermoelectric Heater/Cooler Sandwiched Between a Laser Diode and a Heat Sink/Mass.

\section{Temperature Compensation}

The present sensor was designed to operate over a temperature range of $-65^{\circ} \mathrm{C}$ to $125^{\circ} \mathrm{C}$ with a $\pm 1 \%$ accuracy. The optical fiber coil will function over this range, but because of residual birefringence in the coil and the temperature dependence of the Verdet constant, the sensor will not maintain its calibration over this temperature range. The $\pm 1 \%$ accuracy is accomplished by monitoring the temperature and adjusting the calibration accordingly. The temperature is monitored with a fiber-optic temperature sensor placed near the current sensing coil. Figure 5 shows the temperature sensor, which is based on the change in birefringence of a multiorder waveplate with temperature [11]. The intensity of the light returning from the sensor is proportional to temperature. This light is detected with a photoconductor which is part of an input resistance network of an operational amplifier.
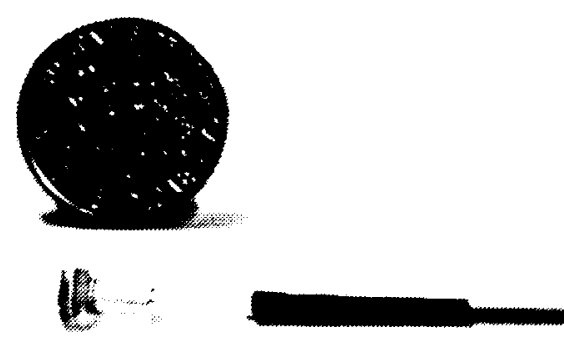

Figure 5 Fiber-Optic Temperature Sensor. 


\section{SENSOR EVALUATION}

\section{Accuracy Over Operating Temperature Range}

The sensor was tested over the range of $-40^{\circ} \mathrm{C}$ to $125^{\circ} \mathrm{C}$ Figure 6 shows a plot of the sensor's performance versus temperature after two temperature cycles. After the two cycles, the slope on the sensor's temperature compensated output versus temperature, from $-10^{\circ} \mathrm{C}$ to $125^{\circ} \mathrm{C}$, was approximately $3.7 \times 10^{-5} /{ }^{\circ} \mathrm{C}$, within the $\pm 1 \%$ calibration. After eight temperature cycles, the low temperature performance dropped so that the sensor held calibration only at temperatures above $20^{\circ} \mathrm{C}$. We believe this is primarily due to the lubricant drying out, i.e., losing its high vapor pressure components. If not for this problem with the lubricant, the sensor would hold calibration over the desired temperature range.

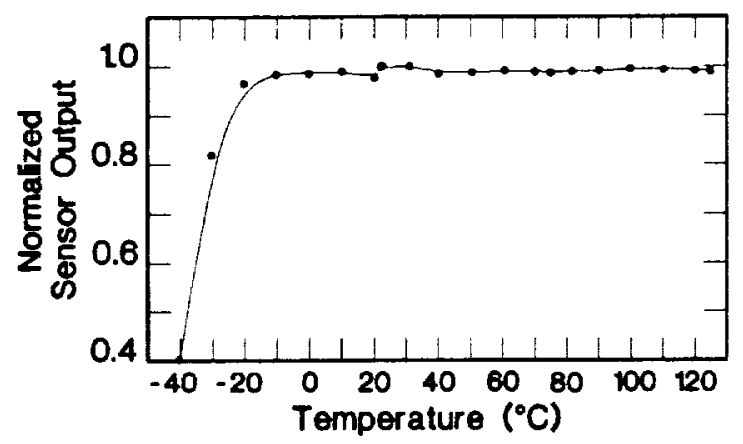

Figure 6 Temperature Compensated Output of the Current Sensor after Two Temperature Cycles.

\section{Noise}

The noise floor or noise equivalent current (NEI) of this sensor is theoretically $0.2 \mathrm{~mA} / \mathrm{VHz}$ with the present detectors and laser [1]. The theoretical NEI calculated by adding the contribution of Johnson and shot noise sources does not include extra amplifier and laser noise which contribute to a higher measured NEI. The measured NEI is approximately $0.6 \mathrm{~mA} / \mathrm{NHz}$. For the measurement of pulse currents the sensor could be operated at its full bandwidth of $1 \mathrm{MHz}$. The NEI for the $1 \mathrm{MHz}$ bandwidth would be approximately 0.6 A/V MHz.

\section{EMI Immunity}

The sensor head is intrinsically EMI immune because it is constructed of all dielectric materials; that is, the sensing head is completely isolated electromagnetically from the opto-electronics package. Only radiated EMI that reaches the diode laser and photodetectors will affect the instrument.

The sensing head responds only slightly to currents that are present outside of the fiber coil. Figure 7 shows a diagram of the isolation which the sensor provides for a current flowing in a wire placed at different locations around the sensing head. The most sensitive location for a current flow outside of the fiber coil is near the middle of the housing limb. The isolation at this location is approximately $-46 \mathrm{~dB}$ relative to a current of the same magnitude flowing inside the fiber coil loop. The reason for this reduction in isolation is not clear, though it occurs at a position closest to where the annealed fiber is spliced to the polarization maintaining fiber and where there is therefore a small gap in closing the sensing loop.

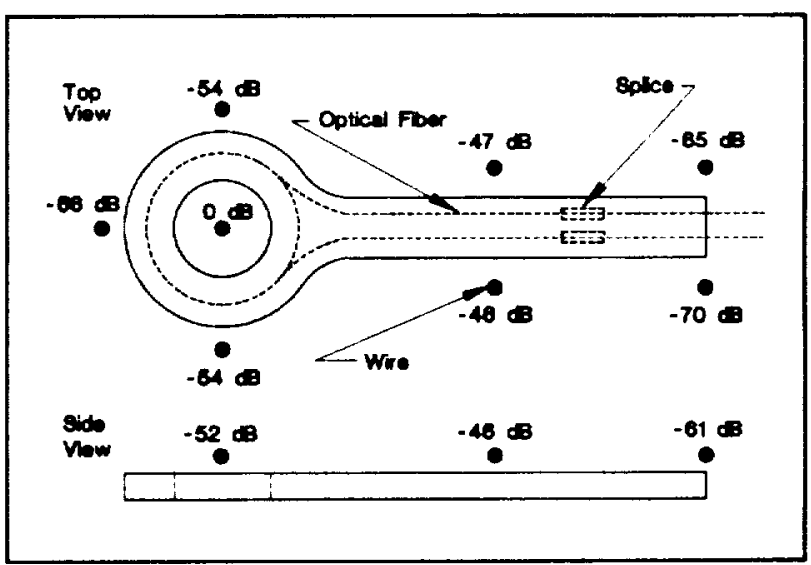

Figure 7 Isolation against Currents Flowing Outside the Sensing Coil.

\section{Vibration Testing}

Only preliminary vibration testing has been done to date. Qualitatively, the sensing head shows some sensitivity to vibration over the frequency range of approximately $30 \mathrm{~Hz}$ to $150 \mathrm{~Hz}$.

\section{Future Tests}

In the near future, NASA will be conducting moreformal vibration tests on the first current sensing unit on vibration testers at the Lewis Research Center. 


\section{SUMMARY}

Figure 8 is a photo of the current sensing head, the optical fiber cable, and the opto-electronics box which contains the optical sources, the optical detectors, and the sensing/compensation electronics.

Presently the fiber optic current sensor can operate between $+20^{\circ} \mathrm{C}$ and $+125^{\circ} \mathrm{C}$ with an accuracy of $\pm 1 \%$ full scale. This accuracy is obtained, in part, by temperature compensation obtained by a fiber optic temperature sensor whose sensing head is buried in the current sensor head.

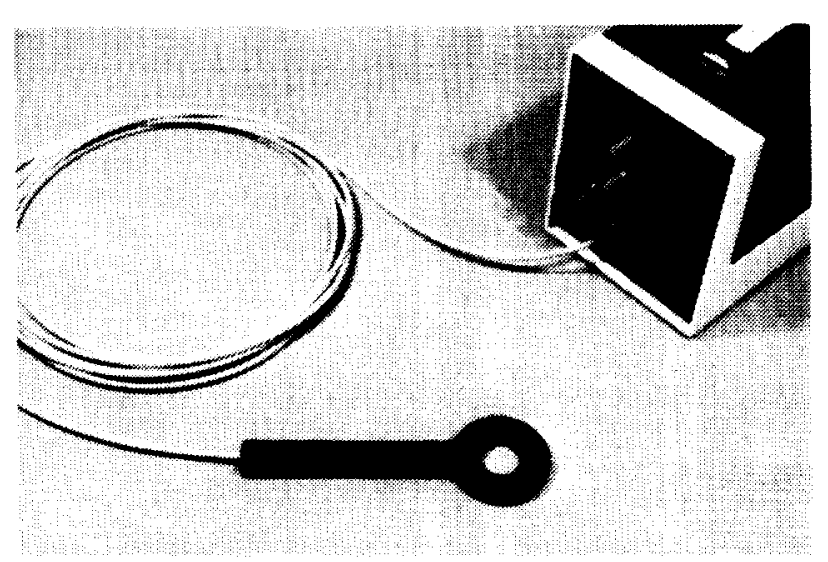

Figure 8 Sensor Head, Fiber-Optic Cables, and Electronics Box.

The present instrument has a calibration of approximately $3.6 \mathrm{mV} / \mathrm{A}$ and a maximum current for $1 \%$ linearity of $720 \mathrm{~A}$.

The prime work for the near future will be to formulate or discover an improved potting grease which will not solidify at low temperature and which will function over the whole desired operating range of $-65^{\circ} \mathrm{C}$ to $+125^{\circ} \mathrm{C}$. A second current sensing head (using the new potting grease) will be built and attached to the present electronics. During 1990 a fiber-optic AC voltage sensor is expected to be completed. Thereafter a ruggedized, field-unit current sensor will be fabricated. The ultimate target of the whole program is the development of a fiber optic power sensor.

\section{ACKNOWLEDGEMENTS}

This work was supported by the NASA Office of Aeronautics and Exploration Technology through the High Capacity Power element of the Civil Space Technology Initiative. Closely related work at NIST has been supported by Los Alamos National Laboratory and the Defense Nuclear Agency. This manuscript represents U. S. Government work and is not subject to copyright.

\section{REFERENCES}

[1] G. W. Day and A. H. Rose, "Faraday effect sensors: The state of the art," Proc. SPIE 985, 138-150, (1988).

[2] D. Tang, A. H. Rose, and G. W. Day, "Practical Considerations in the Design of Optical Current Sensors," Proc. SPIE 1267, (1990).

[3] P. A. Williams, A. H. Rose, G. W. Day, T. E. Milner, and M. N. Deeter, "Temperature dependence of the Verdet constant in several diamagnetic glasses," to be published.

[4] A. J. Rogers, "Optical-fibre current measurement," Int. J. Optoelectronics 3, 391-407, (1988).

[5] Edward A. Ulmer, Jr., "High accuracy Faraday rotation measurements," Technical Digest, Optical Fiber Sensors, New Orleans, 1988.

[6] L. Li, J. R. Qian, and D. N. Payne, "Current sensing using highly birefringent bow-tie fibers," Electron. Lett. 22, 1142-1144, (1986).

[7] R. I. Laming and D. N. Payne, "Electric current sensors employing spun highly birefringent optical fiber," J. Lightwave Technology 7, 2084-2094, (1989).

[8] Z. B. Ren and Ph. Robert, "Input polarization coding in fibre current sensors," in Proc. oth Int Conf. Optical Fiber Sensors, Paris, Sept. 1989.

[9] G. W. Day and S. M. Etzel, "Annealing of bendinduced birefringence in fiber current sensors," Technical Digest, Int. Conf. on Int. Optics and Optical Fiber Commun.--European Conf. Optical Commun., Venice, 1985.

[10] D. Tang, A. H. Rose, and G. W. Day, "Annealing of linear birefringence in single mode fiber coils: Application to optical fiber current sensors," to be published.

[11] T. C. Cetas, "A Birefringent Crystal Optical Thermometer For Measurements of Electromagnetically Induced Heating," Biological Effects of Electromagnetic Waves, Vol. II, 338-348, (1975).

ORIGINAL PAGE IS 


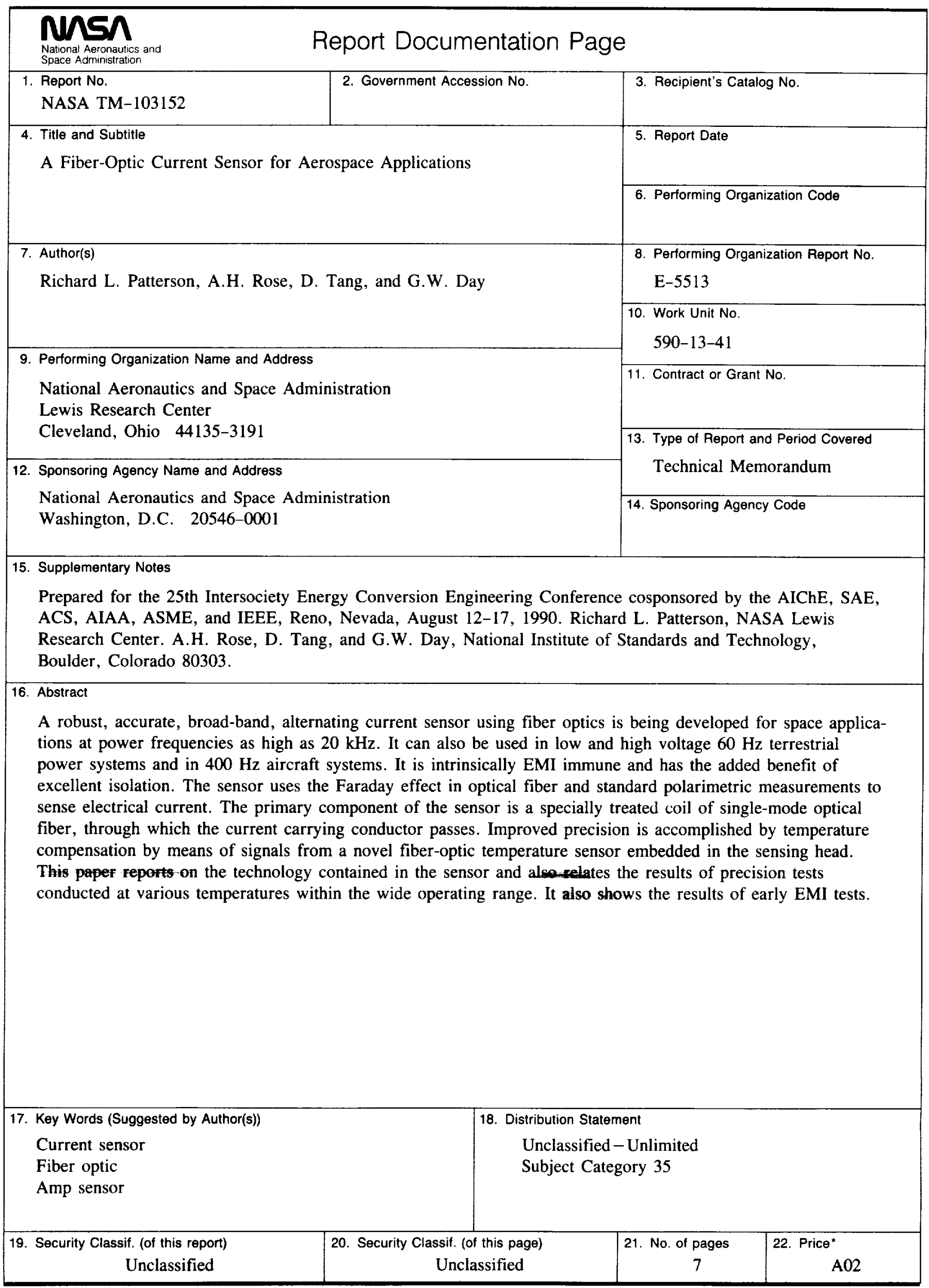

